\title{
Das Gezer-Corpus von El-Amarna: Umfang und Schreiber
}

\author{
von Juan-Pablo Vita - CSIC-Madrid
}

1. Die aus der Stadt Gezer stammenden Briefe bilden eines der bedeutendsten Corpora innerhalb der El-Amarna-Texte ${ }^{1}$. Zu Recht hat man ihnen verschiedene Studien gewidmet, besonders vom linguistischen Standpunkt aus. 1978 legte Sh. Izre'el eine umfassende linguistische Analyse dieser Briefe vor. Das von ihm (1978, 14-15; vgl. auch 1990, 596) abgegrenzte Corpus besteht aus folgenden Briefen: EA 267271, EA 292-294, EA 297-300 und EA 378 (d. h. 13 Briefe). Diese Texte dienten auch als Basis für weitere syntaktische Untersuchungen der Gezer-Briefe durch J. L. Hayes (1984, 55-123) und J. P. van der Westhuizen (1995). Kürzlich schlug Sh. Izre'el (1995, 109-118) vor, daß auch der Brief EA 369 (vom Pharao an Milkilu) eventuell zu diesem Corpus gehört.

2. Die unter $\S 1$. aufgezählten Texte bilden aber sehr wahrscheinlich nicht die Gesamtmenge der Gezer-Briefe. Auf diese Tàtsache hat schon J. A. Knudtzon im Kommentarband seiner Amarna-Textausgabe aufmerksam gemacht, und zwar in folgenden Beobachtungen:

„Die Absenderin von Nr. 273 und 274 steht nach dem Inhalt ihrer Briefe (vgl. bes. 273, $23 \mathrm{f}$.) höchstwahrscheinlich in irgendwelcher Verbindung mit Milkilu, wofür auch spricht, daß ihre Briefe nach Schrift und Ton denen Milkilus (267-271) gleich sind. Dies gilt allerdings auch von 272 [auf S. 1328 Anm. 1: ,Nach Schrift und Ton den MilkiluBriefen (267-271) gleich'] und 275-280. Die Form der Tafel 273 ist

1 Dieser Artikel wurde während eines Forschungsaufenthaltes am Altorientalischen Seminar der Freien Uniyersität Berlin mit Unterstützung der. Alexander von Humboldt-Stiftung erarbeitet. Ziel des Projektes, das im Rahmen der im CSIC (Madrid) hergestellten Philologischen Datenbank des Nordwestsemitischen (BDFSN. Dir.: Prof. Dr. J.-L. Cunchillos) durchgeführt wird, ist die Erstellung einer elektronischen Datenbank des El-Amarna-Corpus. Mein Dank gilt Prof. Dr. J. Renger (Freie Universität Berlin) für die freundliche Aufnahme und Hilfsbereitschaft, sowie PD Dr. J. Tropper (Humboldt-Universität Berlin) für Diskussion und Anregungen zum Manuskript sowie für seine Mithilfe bei der deutschen Fassung dieses Beitrags. 
ganz wie die von 271, doch die Grösse etwas verschieden" (Knudtzon 1907-1915, 1328 Anm. 2) ${ }^{2}$.

„Die Tafeln Nr. 275-277 sind nach Schrift und Ton einander und Nr. 267-274 ebenso wie 278-280 gleich. Nach dem was 276,4 vom Namen des Absenders zu sehen ist, wird dieser derselbe sein wie der von 275. Ob aber 277 von demselben Manne herrührt wie 275 und 276, was ich einst annahm, muss dahingestellt bleiben“" (Knudtzon 1907-1915, 1329 Anm. 1).

„Die Briefe des Šuwardata zerfallen ... nach ihrer Schrift in zwei Klassen: a) Nr. 278-280, welche dieselbe Schrift haben wie die MilkiluTafeln und andere oben erwähnte, die mit diesen übereinstimmen; b) Nr. 281-284, die einen anderen Schrifttypus aufweisen. Ganz gleiche Schrift finden wir jedoch nicht auf den letztgenannten" (Knudtzon 1907-1915, 1329 Anm. 2).

Knudtzon zufolge rühren also die Briefe EA 272-280 von demselben Schreiber her, der auch die Briefe 267-271 verfaßt hat, d. h. vom Schreiber Milkilus, des Herrschers von Gezer. Auch sie wären folglich dem Gezer-Corpus zuzuordnen. W. L. Moran (1987, 500 Anm. 1; 1992, 318 Anm. 6, 320 Anm. 1) unterstützte diese Auffassung in seiner Bearbeitung der Amarnabriefe.

2.1. Im Zuge der Kollationierung der Berliner Amarnabriefe ${ }^{3}$ konnte ich mich nicht nur von der Richtigkeit der angeführten Beobachtungen Knudtzons überzeugen, sondern auch eine weitere Tafel identifizieren, die ebenfalls dem Schreiber Milkilus zuzuordnen ist. Es handelt sich um den Brief EA 266 (VAT 1590; Abb. I), der bisher in diesem Zusammenhang unbemerkt geblieben ist (vgl. § 3.1. und § 4.3.). Die Überprüfung dieser Tatsache kann durch die Beobachtung einiger schon publizierter Photos erleichtert werden; $\mathrm{m}$. W. gibt es zu folgenden Briefen Photos: EA 270 (BB pl. 2), EA 278 (BB pl. 5; Smith 1998, 158), EA 279 (Smith 1998, 158-159), EA 294 (BB pl. 1), EA 297 (BB pl. 5), EA 299 (Barnett 1977, 14) 4 .

2.2. Folgende Briefe lassen sich also vorläufig dem Gezer-Corpus zuordnen: EA 266-280, 292-294, 297-300 und 378. Es handelt sich

2 Die Kursive der in diesem Artikel vorkommenden Knudtzon-Zitate stammt von uns.

3 Dr. J. Marzahn (Vorderasiatisches Museum) sei hier für die Erlaubnis zur Kollationierung der Berliner Amarna-Briefe sowie für seine Hilfsbereitschaft herzlich gedankt.

4 Die von O. Schroeder (1915) hergestellten Kopien der Gezer-Briefe aus dem Berliner Vorderasiatischen Museum geben auch einen ziemlich genauen Eindruck der Schrift dieser Tafeln wieder. 
somit um insgesamt 23 Briefe (24 mit EA 369, vgl. $\S 1.)^{5}$, von denen 15 vom gleichen Schreiber verfaßt sind (EA 266-280). Das Corpus als Ganzes dürfte aber noch größer sein (vgl. §4.).

3. Ein einziger Schreiber verfaßte also mindestens 15 der aus Gezer erhaltenen Briefe, d. h. fünf Briefe Milkilus (EA 267-271) und zehn weitere von verschiedenen anderen Stadtfürsten (EA 266, EA 272280). Im folgenden soll insbesondere nach den historischen Umständen gefragt werden, die erklären könnten, warum ein bestimmter Schreiber aus der Verwaltung Milkilus, des Herrschers von Gezer, auch für Fürsten außerhalb dieser Stadt tätig war.

3.1. Der Brief EA 266 wurde von Tagi, dem Fürsten von Gintikirmil (EA 289), an den Pharao gesandt. Insgesamt gibt es drei Briefe, EA 264, 265 und 266, die Tagi als Absender nennen. Es wurde aber schon von Knudtzon (1907-1915, 828 Anm. a; 1323 Anm. 2) bemerkt, daß EA 266 eine andere Schrift aufweist als die anderen zwei Briefe. Offensichtlich wurde EA 266 - anders als EA 264 und 265 - vom Schreiber der Milkilu-Briefe verfaßt, wie aus dem Schriftduktus eindeutig hervorgeht (siehe Abb. I). Diese Tatsache erklärt ebenfalls den von R. S. Hess bemerkten Unterschied in den Schreibungen des Namens Tagi zwischen EA 264-265 einerseits (Ta-gi) und EA 266 anderseits $(T a-a-g[i])^{6}$. Die enge persönliche Beziehung zwischen Tagi und Milkilu ist aus anderen Amarnabriefen deutlich zu erkennen: Tagi ist der Schwiegervater Milkilus (EA 249), und beide sind politisch und militärisch verbündet (EA 289). So läßt sich erklären, warum ein Schreiber voin Milkilu gelegentlich auch Briefe für Tagi schreiben durfte bzw: mußte.

3.2. Die Fürstin von Sapuma, NIN-UR.MAH.MEš, ist die Absenderin der Briefe EA 273 (Abb. II) und 274. In EA 274 berichtet sie dem Pharao, daß Șapuma durch die 'apīru-Leute erobert worden sei. Es liegt auf der Hand, daß die Fürstin diesen Brief nicht aus ihrer Stadt abgeschickt hat. Die Identifizierung des Schreibers beider Briefe als desjenigen von Milkilu ermöglicht die Folgerung, daß die Fürstin, nach Eroberung von Sapuma, in Gezer Zuflucht fand und ihre Briefe am Hofe von Milkilu schreiben und absenden ließ. Diese Tatsache setzt gute politische Beziehungen zwischen beiden Städten voraus und könnte ein Beweis dafür sein, daß Șapuma, dessen Lage unbekannt bleibt? ${ }^{7}$, nicht weit von Gezer entfernt zu lokalisieren ist.

\footnotetext{
5 Zur möglichen Zuordnung von EA 300 zum Gezer-Corpus siehe Izre'el 1978, 1415 (Anm. 15).

6 Hess 1993, 154.

7 Vgl. Moran 1987, 601; 1992, 391.
} 


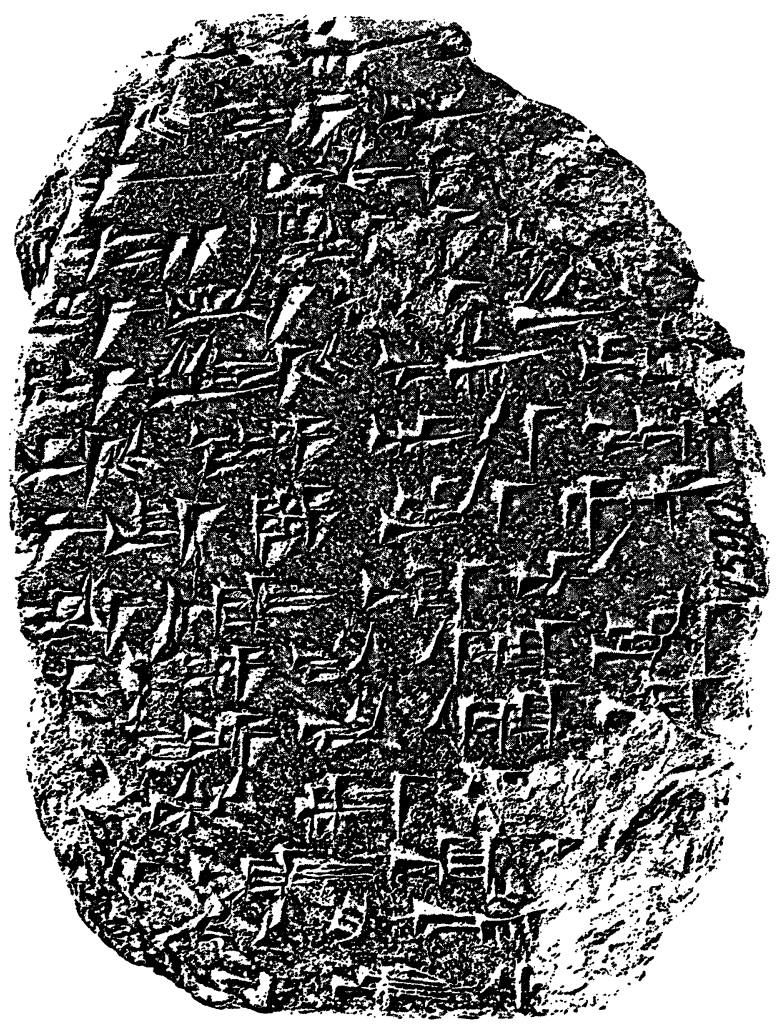

Abb. I: EA 266 


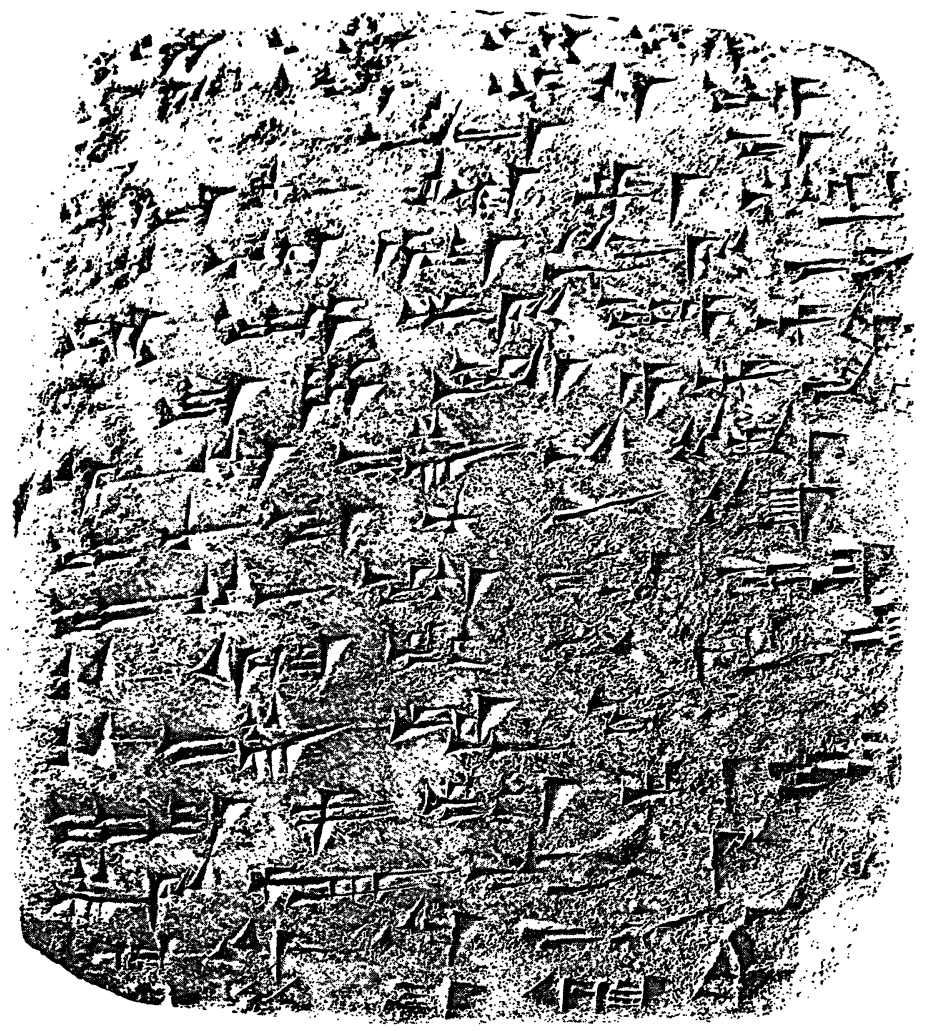

Abb. II: EA 273 
3.3. Die Briefe EA 275, 276, 277 und 278 weisen unterschiedliche Absender auf (erhalten sind Yahzib-Adda [EA 275-276] und Šuwardata [EA 278]), ihre Inhalte aber sind wörtlich identisch: ${ }^{8}$ Es heißt dort, die betreffenden Stadtfürsten würden den Befehl, den sie vom Pharao erhalten hatten, tatsächlich durchführen. Schon allein der identische Inhalt legt die Annahme nahe, daß diese Briefe das gleiche Ziel verfolgten und gleichzeitig verfaßt wurden ${ }^{9}$. Die Feststellung, daß alle vier Briefe von Milkilus Schreiber herrühren, stützt nicht nur diese Hypothesen, sondern zeigt auch, daß die Briefe aus der Verwaltung Gezers stammen. Dieser Tatbestand beweist somit, zumindest für diese bestimmte Zeit, daß zwischen Milkilu, Šuwardata, Yahzib-Adda und dem Fürsten von EA 277 gute politische Beziehungen bestanden ${ }^{10}$.

3.4. Der Brief EA 278 (vgl. § 3.3.) wiederum zeugt von guten Beziehungen zwischen Šuwardata, der möglicherweise Herrscher von Qiltu (vgl. EA 279) war ${ }^{11}$, und Milkilu von Gezer. Zwei weitere Briefe, einer von Milkilu selbst (EA 271) und einer ihres gemeinsamen Feindes 'Abdi-Heba von Jerusalem (EA 290), bestätigen, daß beide Herrscher zu einer bestimmten Zeit Verbündete waren. Der Rest der ŠuwardataBriefe $^{12}$ berichtet von kriegerischen Umständen, die Šuwardata ständig bedrohten.

3.5. Vor dem Hintergrund des Bündnisses zwischen Šuwardata und Milkilu einerseits und des Krieges gegen Šuwardata anderseits sollten auch die Šuwardata-Briefe EA 279 und 280, die beide aus der Verwaltung Milkilus stammen, gelesen werden. In EA 279 äußert Šuwardata seine Absicht, gegen Qiltu vorzurücken; er befand sich damals also außerhalb seines Territoriums. In EA 280 dagegen hat er Qiltu bereits zurückerobert. Das heißt, während die Ereignisse von EA 279 und 280 stattfanden, befand sich Šwardata im Gebiet von Gezer und berichtete von dort dem Pharao ${ }^{13}$.

4. Die Tatsache, daß die Verwaltung von Gezer während der Regierung Milkilus auch für andere Fürsten tätig war ${ }^{14}$, bietet also eine befriedigende Erklärung der historischen Umstände seiner Zeit. Welche Folgerungen sind daraus zu ziehen?

8 Wie von Moran (1992, 320 Anm. 1) schon angemerkt.

9 Vgl. schon Campbell 1964, 112-113.

10 Es gibt innerhalb des Amarna-Corpus weitere Briefe gleichen Inhalts, die von verschiedenen Absendern stammen; vgl. EA 174, 175, 176 und 363.

11 Zur Diskussion siehe Hess 1993, 151.

12 Siehe zu diesem Corpus Smith 1998, 125-170; vgl. auch weiter § 5.4.

$13 \mathrm{Vgl}$. in diesem Sinne auch Moran 1987, 500 Anm. 1.

14 Siehe zu diesem Punkt auch Izre'el 1977, 163. 
4.1. Nebst dem Jerusalem-Schreiber (Moran 1975) ist Milkilus Schreiber mit fünfzehn von ihm verfaßten Briefen (EA 266-280) ${ }^{15} \mathrm{der}$ zur Zeit am besten identifizierte Schreiber innerhalb des El-AmarnaCorpus. Die Anzahl der Briefe, die ihm und folglich dem Gezer-Corpus zuzuschreiben sind, dürfte aber noch größer sein. Der Brief EA 266 bietet dafür Anlaß zur Diskussion.

4.2. EA 266 kommt eine besondere Stellung innerhalb der Briefe des Schreibers von Milkilu zu. Er weicht wegen seiner langen und hochpoetischen Einleitung deutlich von den übrigen Gezerbriefen ab. Der Grund dafür dürfte darin liegen, daß der Brief für einen anderen Herrscher, nämlich für Tagi von Gintikirmil, geschrieben wurde; Tagi hatte sich sozusagen einen besonderen Inhalt gewünscht. Die Formulierung dieses Briefes, einschließlich der sogenannten „Ziegel-Formel"16, weist aber deutliche Übereinstimmungen nicht nur mit den anderen Briefen von Tagi (EA 264, 265) auf, sondern auch mit EA 292 (von ${ }^{\text {IM-DI.KUD }}{ }^{17}$ von Gezer) und EA 296 (von Yahtiru, [möglicherweise] Herrscher eines unbekannten Ortes) ${ }^{18}$. Diese durch die Formulierungen bestehende Verbindung wird durch folgende epigraphische Bemerkungen Knudtzons gestützt:

„Die Tafeln 292-294 scheinen ganz dieselbe Schrift zu haben ... Die Schrift findet sich auch auf 296 und 297, und ist derjenigen der Milkilu-Tafeln usw. ähnlich, nicht aber in allen Einzelheiten gleich (so weichen el, en und šêpu etwas ab). Inhaltlich gehören 292 und 294 zusammen" (Knudtzon 1907-1915, 1344 Anm. 2).

„Die Tafel [EA 296] stimmt in bezug auf Schrift mit 292-294 und in bezug auf Ton mit 294 überein“ (Knudtzon 1907-1915, 1346 Anm. 1).

„Nr. 297, die von einem Iapahi herrührt, weist dieselbe Schrift auf wie 292-294 und 296 und wird in bezug auf den Ton Nr. 292 und 293 gleich sein" (Knudtzon 1907-1915, 1346 Anm. 2).

Diesen Bemerkungen zufolge sind die Briefe EA 292-294, 296 und 297 dem Schreiber Milkilus zuzuschreiben. Wenn man die veröffentlichten Photos einiger dieser Tafeln überprüft ${ }^{19}$, sieht man die Genau-

15 Er schrieb sowohl für sèinen Herrn als auch für andere Herrscher (Tagi, NIN-UR.MAH.MEŠ, Yahzzib-Adda, Šuwardata und die Absender von EA 272 und EA 277).

16 Siehe dazu Rainey 1996 (Bd. III) 64; CAD L 176.

17 Zur Diskussion des Namens siehe Hess 1993, 53.

18 Die genaue inhaltliche Beziehung dieser Briefe wurde von Weber (teilweise) (1915, 1324) und Moran (1987, 522 Anm. 2; 1992, 335 Anm. 2) anerkannt.

19 EA 294: BB pl. 1; EA 297: BB pl. 5. Das von Moran (1987, 526; 1992, 338) zitierte Werk von C. Pfeiffer, Tell el Amarna and the Bible (1963), das auf Seite 11 ein Photo der Rückseite von EA 296 enthält, ist mir nicht zugänglich gewesen. 
igkeit der epigraphischen Bemerkungen Knudtzons bestätigt und stellt fest, daß die Schrift dieser Tafeln beinahe völlig mit der des MilkiluSchreibers übereinstimmt und nur in wenigen Details davon abweicht.

4.3. Könnte es sich tatsächlich um denselben Schreiber handeln? Beim Versuch, diese Frage zu beantworten, muß die Chronologie der betreffenden Briefe berücksichtigt werden. Die Briefe, die mit Sicherheit von Milkilus Schreiber verfaßt wurden, d. h. EA 266-280, umfassen die Zeitspanne der letzten Regierungsjahre von Amenophis III. und der ersten Jahre von Amenophis IV. ${ }^{20}$ Während EA 296 auch in diese Epoche gehört (Campbell 1964, 100. 134), sind die anderen vier (EA 292-294, 297) in die Mitte der Regierungszeit von Amenophis IV. zu datieren (Campbell 1964, 126. 135). Es wäre also nicht unmöglich, daß alle diese Briefe das Werk eines einzigen Mannes darstellen. Falls. dies zutrifft, wäre dieser Schreiber nicht nur für einen einzigen Herrscher von Gezer tätig gewesen, sondern mindestens für drei: für Milkilu (EA 266-280), für ${ }^{d}$ IM-DI.KUD (EA 292-294) ${ }^{21}$ und für Yapahu (EA 297) ${ }^{22}$. Die wenigen epigraphischen Varianten, die in den jüngeren Texten zu beobachten sind, könnten als persönliche Entwicklung des Schreibers zu betrachten sein. Sollten aber weitere Untersuchungen beweisen, daß es sich um zwei verschiedene Schreiber handelt, müßte man von einer echten „Gezer-Schreiberschule“ sprechen ${ }^{23}$.

5. Wir gelangen also zu folgendem vorläufigen Resultat, was den Umfang des Gezer-Corpus und seine(n) Schreiber betrifft:

5.1. Folgende 24 Briefe sind dem Gezer-Corpus zuzuordnen: EA 266-280, 292-294, 296-300, 378. Ein weiterer Brief, der sehr wahrscheinlich hierher gehört, ist EA 369 (vgl. § 1.). Das Gezer-Corpus würde demnach 25 Briefe umfassen.

5.2. Folgende 15 Briefe sind mit Sicherheit Milkilus Schreiber zuzuordnen: EA 266-280. Als weitere Briefe, die eventuell von Milkilus Schreiber verfaßt wurden, kommen in Betracht: EA 292-294, 296 und 297. Dieser Schreiber hätte somit insgesamt 20 Briefe verfaßt. Die letztgenannten Briefe könnten aber eventuell auch von einem anderen,

20 Den „First Chronological Focal Point“" von Campbell 1964; siehe ibid. zu den einzelnen Briefen und S. 134.

$21 \mathrm{Zu}$ EA 293 als Brief von diM-DI.KUD siehe Moran 1987, 522 Anm. 1; 1992, 336 Anm. 1; Na'aman 1997. Zu EA 294 vgl. Moran 1987, 523. 524 Anm. 1; 1992, 336. 337 Anm. 1.

22 Diese Tatsache würde dem Vorschlag von van der Westhuizen (1995, 14), daß jeder neue Herrscher seine(n) eigenen Schreiber ernannte, widersprechen.

$23 \mathrm{Zu}$ dieser Annahme siehe schon Izre'el 1977, 160. 163, in bezug auf EA 292 und 294. 
mit dem Schreiber Milkilus eng verwandten Schreiber herrühren. Alle Briefe wären aber das Produkt der „Gezer-Schule“.

5.3. Was den Rest der Gezer-Briefe betrifft, so beschreibt Knudtzon (1907-1915, 1346 Anm. 2): „298-300 gleichen einander sowohl in Schrift als in Ton und sind nach beiden mit einer Reihe der Nummern $301 \mathrm{ff}$. zusammenzustellen“. EA 378 wurde vom gleichen Schreiber erstellt, wie schon Millard $(1965,143)$ feststellen konnte. Ein dritter Schreiber kommt als Verfasser von EA 369 in Betracht ${ }^{24}$. Sollten, wie unter $\$ 5.2$. vorgeschlagen, zwanzig Briefe dem Milkilu-Schreiber zuzuschreiben sein, würden alle Gezer-Briefe von nur drei Schreibern stammen.

5.4. Das Šuwardata-Corpus seinerseits besteht aus folgenden Briefen: EA 278-284 und 366. Von diesen wurden EA 278-280 vom Schreiber Milkilus verfaßt und sind so zum „Gezer-Dialekt“ zu stellen. Die übrigen fünf Šuwardata-Briefe wurden von zwei bzw. drei weiteren Schreibern verfa $3 t^{25}$.

5.5. Zukünftige Forschungen könnten dieses Gesamtbild noch modifizieren und verfeinern. Es ist nicht auszuschließen, daß man in $\mathrm{Zu}$ kunft noch weitere zum Gezer-Corpus gehörige Briefe identifizieren kann.

6. Wir kommen somit zu den folgenden Schlußfolgerungen:

6.1. Das hier neu abgegrenzte, gewachsene Gezer-Corpus umfaßt, wie unter $\S 5.1$. erwähnt, 24 bzw. 25 Briefe. Es ist festzustellen, daß Gezer auf diese Weise das zweitgrößte Corpus innerhalb der AmarnaBriefe überhaupt bildet (das größte, mit 69 Briefen, bleibt Byblos). Als Folge ergibt sich, daß andere Corpora zusammenschrumpfen bzw. samt ihrem angeblichen „Dialekt“ ganz verschwinden. Konkret ist der „Šuwardata-Dialekt" nicht mehr in acht Briefen, wie bisher angenommen wurde ${ }^{26}$, bezeugt, sondern nur noch in fünf (EA 281-284, 366), und es gibt keine Briefe aus Sapuma mehr ${ }^{27}$. Folglich kann von keinem „Șapuma-Dialekt“ mehr die Rede sein.

6.2. Die wertvollen, unter $\S 1$. erwähnten Abhandlungen zum Gezer-Dialekt betrachten also nicht die Gesamtheit der Gezer-Texte. Andere linguistische Werke dagegen grenzen die von ihnen untersuchten Corpora falsch $a^{28}$. Das Gezer-Corpus und die Suwardata-Briefe müssen folglich philologisch und historisch weiter untersucht werden.

24 Dazu ausführlich Izre'el 1995, 109-118.

25 Vgl. dazu Knudtzon 1915, 1329 Anm. 2; Moran 1992, 320 Anm. 1.

26 Vgl. u. a. kürzlich Smith 1998.

27 Bisher galten EA 273 und EA 274 als Briefe aus Sapuma; siehe § 3.2.

28 Vgl. etwa Smith 1998. 
6.3. Abschließend sei betont, daß der Name des Absenders eines Briefes allein kein entscheidendes Kriterium für die linguistische Abgrenzung der Amarna-Corpora darstellt. Briefe eines bestimmten Stadtfürsten können ein Produkt seiner eigenen Verwaltung sein oder von der Verwaltung eines mit ihm befreundeten bzw. alliierten Fürsten stammen. Da die Amarna-Dialekte streng nach Corpora zu untersuchen sind, müssen diese zuerst so sorgfältig wie möglich nach Schreibern abgegrenzt werden 29 . Im Falle von Gezer z. B. müßte die Untersuchung der von den einzelnen Gezer-Schreibern (vgl. § 5.2.-5.3.) verwendeten Sprache die Grundlage für die Beschreibung des gesamten Gezer-Dialekts bilden. Die linguistische Abgrenzung der Amarna-Corpora nach Schreibern ermöglicht neue Einblicke in die politischen Beziehungen zwischen den syro-palästinischen Staaten dieser Zeit und kann so zu ei-. nem besseren Verständnis der historischen Ereignisse beitragen.

\section{Literatur und literarische Abkürzungen}

R. Barnett 1977: Illustrations of Old Testament History 2 - - B B $=$ C. Bezold/E. W. Budge 1892: The Tell el-Amarna Tablets in the British Museum.

E. F. Campbell 1964: The Chronology of the Amarna Letters.

J. L. Hayes 1984: Dialectal Variation in the Syntax of Coordination and Subordination in Western Akkadian of the el-Amarna Period (PhD Diss., UCLA). - R. S. Hess 1993: Amarna Personal Names.

Sh. Izre'el 1977: Two Notes on the Gezer-Amarna Tablets, Tel Aviv 4, 159-167. ders. 1978: The Gezer Letters of the El-Amarna Archive. Linguistic Analysis, Israel Oriental Studies 8, 13-90. - ders. 1990: A New Translation of the Amarna Letters, BiOr. 47, 577-604. - ders. 1995: The Amarna Glosses: Who Wrote What for Whom? Some Sociolinguistic Considerations, Israel Oriental Studies 15, 101-122.

J. A. Knudtzon 1907-1915: Die El-Amarna-Tafeln (=VAB 2).

A. Millard 1965: A Letter from the Ruler of Gezer, Palestine Exploration Quarterly 97, 140-143. - W. L. Moran 1975: The Syrian Scribe of the Jerusalem Amarna Letters, in (ed.) H. Goedicke/J. J. M. Roberts, Unity and Diversity, 146-166. - ders. 1987: Les lettres d'El Amarna. - ders. 1992: The Amarna Letters.

N. Na'man 1997: EA 293, NABU 1997:121.

A. F. Rainey 1996: Canaanite in the Amarna Tablets (4 vol.).

O. Schroeder 1915: VS 11-12. - S. P. Smith 1998: The Inflectional Morphology of the yvqtvl-Verb in the Šuwardata Amarna Letters (EA 278-284, 366), in: (ed.) Sh. Izre'el/1. Singer/R. Zadok, Past Links. Studies in the Languages and Cultures of the Ancient Near East (Israel Oriental Studies 18), 125-170.

O. Weber 1915: Anmerkungen zu J. A. Knudtzon, Die El-Amarna-Tafeln, in: Knudtzon 1907-1915, 1009-1357. - J. P. van der Westhuizen 1995: Word Order Variation of Verbal Sentences in Selected Gezer Amarna Letters, Journal for Semitics 7, $1-15$.

29 Wie wichtig für diesen Zweck die epigraphischen Anmerkungen Knudtzons immer noch sind, sei hier nochmals hervorgehoben. Man beachte in diesem Zusammenhang auch die Erwägungen von Izre'el (1990, 590-591). 